\title{
Review
}

\section{Antibiotic resistance trends and mechanisms in the foodborne pathogen, Campylobacter}

\author{
Yizhi Tang ${ }^{1}$, Liangxing Fang ${ }^{1,2}$, Changyun $\mathrm{Xu}^{1}$ and Qijing Zhang ${ }^{1 *}$ \\ ${ }^{1}$ Departments of Veterinary Microbiology and Preventive Medicine, Ames, IA, USA \\ ${ }^{2}$ National Risk Assessment Laboratory for Antimicrobial Resistance in Bacteria of Animal Origin, \\ South China Agricultural University, Guangzhou, China
}

Received 17 August 2017; Accepted 6 October 2017; First published online 23 November 2017

\begin{abstract}
Campylobacter is a major foodborne pathogen and is commonly present in food producing animals. This pathogenic organism is highly adaptable and has become increasingly resistant to various antibiotics. Recently, both the Centers for Disease Control and Prevention and the World Health Organization have designated antibiotic-resistant Campylobacter as a serious threat to public health. For the past decade, multiple mechanisms conferring resistance to clinically important antibiotics have been described in Campylobacter, and new resistance mechanisms constantly emerge in the pathogen. Some of the recent examples include the $\operatorname{erm}(B)$ gene conferring macrolide resistance, the $\operatorname{cfr}(C)$ genes mediating resistance to florfenicol and other antimicrobials, and a functionally enhanced variant of the multidrug resistance efflux pump, CmeABC. The continued emergence of new resistance mechanisms illustrates the extraordinary adaptability of Campylobacter to antibiotic selection pressure and demonstrate the need for innovative strategies to control antibiotic-resistant Campylobacter. In this review, we will briefly summarize the trends of antibiotic resistance in Campylobacter and discuss the mechanisms of resistance to antibiotics used for animal production and important for clinical therapy in humans. A special emphasis will be given to the newly discovered antibiotic resistance.
\end{abstract}

Keywords: Campylobacter, antibiotic resistance, multidrug efflux pump, food safety.

\section{Introduction}

Campylobacter species, particularly Campylobacter jejuni and Campylobacter coli, are a major cause of foodborne bacterial gastroenteritis in humans (Ruiz-Palacios, 2007). As estimated by the Centers for Disease Control and Prevention (CDC), Campylobacter is responsible for 1.3 million cases of foodborne illnesses annually in the USA (Scallan et al., 2011). It was also estimated that Campylobacter spp. are responsible for 400-500 million cases of diarrhea each year worldwide (Ruiz-Palacios, 2007). Transmission of Campylobacter to human beings occurs mainly through contaminated food of animal origin, particularly raw or undercooked poultry meat, unpasteurized milk, and dairy products (Allos, 2001; Stanley and Jones, 2003; CDC, 2009).

*Corresponding author. E-mail: zhang123@iastate.edu
Although the majority of Campylobacter infections are self-limited, do not require antimicrobial treatment, and usually resolve within a few days without antibiotic treatment, severe or prolonged infection may occur, particularly in the young, elderly, and individuals with compromised immunity (Allos, 2001). In these circumstances, fluoroquinolone (FQ) and macrolide antibiotics are the drugs of choice for treatment (Allos, 2001; Engberg et al., 2001). Intravenous administration of aminoglycosides are only used for the treatment of serious bacteremia and other systemic infections due to Campylobacter (Aarestrup and Engberg, 2001). Beta-lactam is not recommended for treatment of campylobacteriosis, but oral beta-lactam, such as co-amoxiclav, might be an appropriate agent when Campylobacter isolates are resistant to both FQ and macrolides (Elviss et al., 2009; Griggs et al., 2009).

As a foodborne pathogen, Campylobacter is prevalent in the intestinal tracts of food producing animals and is frequently exposed to antibiotics used for animal production. In response 
to the selection pressure from antibiotics used for animal agriculture and human medicine, Campylobacter has evolved various mechanisms for resistance to clinically important antibiotics. Both the CDC and the World Health Organization have recently listed drug-resistant Campylobacter as a serious antibiotic resistance threat (CDC, 2013; WHO, 2017). Because of the importance of Campylobacter in food safety and public health, many studies have been performed to understand the epidemiology and mechanisms of antibiotic resistance in this organism. This review will summarize the current knowledge of antibiotic resistance in Campylobacter, with an emphasis on clinically important and newly discovered antibiotic-resistance mechanisms.

\section{Trends of antibiotic resistance in Campylobacter}

FQ antimicrobials were first introduced for clinical therapy and animal production in the 1980s, and FQ-resistant Campylobacter was initially reported in the late 1980s in Europe (Engberg et al., 2001). Since then, a drastic increase in the incidence of FQ-resistant Campylobacter has been reported in different countries worldwide (Padungton and Kaneene, 2003; Luangtongkum et al., 2009; Nguyen et al., 2016; Sierra-Arguello et al., 2016; Wozniak-Biel et al., 2017). Several studies have also linked the use of FQs with the emergence and spread of FQ-resistant Campylobacter (Endtz et al., 1991; van Boven et al., 2003; Humphrey et al., 2005). In the USA, the introduction of sarafloxacin and enrofloxacin in the mid-1990s, for use in poultry, was linked to the rise of FQ-resistant Campylobacter (Nachamkin et al., 2002). Although FQs were used for the control of respiratory disease and were not intended for control of Campylobacter in poultry, the unintended consequence of this usage is the selection of FQ-resistant Campylobacter, which is commonly present in the intestinal tract of birds (McDermott et al., 2002; Luo et al., 2003; Zhang et al., 2003). One study indicated that before 1992 FQ-resistant C. jejuni was rarely observed in the USA, whereas from 1992 to 2001, FQ-resistant C. jejuni of human origin increased from 1.3 to 40.5\% (Nachamkin et al., 2002). A similar rising trend in FQ resistance among Campylobacter isolates was also reported in other countries. For example, ciprofloxacin resistance among Campylobacter species from humans increased from zero before 1991 to $84 \%$ in 1995 in Thailand (Hoge et al., 1998). A study across 17 years showed that the rates of ciprofloxacin resistance of clinical C. jejuni isolated in China increased from 50\% to $93.1 \%$ between 1994 and 2010 (Zhou et al., 2016). A recent study from China found that almost $100 \%$ of the C. jejuni and C. coli isolates from chicken and swine were resistant to FQs (Wang et al., 2016). In Spain, FQ resistance among clinical Campylobacter isolates was not observed in 1987; however, in 1991 the frequency of FQ-resistant Campylobacter strains had increased remarkably to 30\% (Endtz et al., 1991). Additionally, a steady increase in FQ-resistance among Campylobacter isolates has also been observed in many European countries (Lucey et al., 2002; Pezzotti et al., 2003; Gallay et al., 2007; Nguyen et al., 2016).

Compared with FQ resistance, macrolide resistance is much less prevalent in Campylobacter. However, increased but varied prevalence of macrolide-resistant $C$. jejuni and $C$. coli has been reported in both developed and developing countries (Wang et al., 2016). In most developed countries, macrolide resistance is $<10 \%$ (Engberg et al., 2001; Cha et al., 2016), significantly lower than FQ resistance. In the USA, the NARMS (National Antimicrobial Resistance Monitoring System) 2014 report indicated that erythromycin resistance in the $C$. jejuni isolates from both human and chicken sources was $<2 \%$, which is lower than in C. coli (around 10\%). Studies conducted by the National Animal Health Monitoring System (NAHMS) Dairy 2002 and Dairy 2007 reported that $0.4 \%$ of the cattle Campylobacter isolates were resistant to erythromycin (USDA, 2011). Similar findings also were observed in European countries, where macrolides resistance among Campylobacter isolates from human and C. jejuni isolates from chicken and cattle has been low and stable (Gibreel and Taylor, 2006; Papavasileiou et al., 2007; Bardon et al., 2009). However, in the case of Campylobacter isolates of animal origin from some developing countries, high prevalence of macrolide resistance, especially in C. coli from poultry and swine, has been reported in multiple studies (Li et al., 2016; Shobo et al., 2016; Singh and Mittal, 2016; Wang et al., 2016). This may be related to the use of macrolide agents for prevention and control of animal diseases. Interestingly, many studies have found that macrolide-resistant C. coli is much more prevalent than macrolide-resistant C. jejuni (Li et al., 2016; Shobo et al., 2016; Wang et al., 2016). For example, a recent report from China indicated that $<10 \%$ of C. jejuni isolated from human, chicken and swine hosts were resistant to macrolides, while up to $73.2 \%$ of $C$. coli isolates were resistant to the antibiotics (Wang et al., 2016). The exact reason for the much higher prevalence of macrolide resistance in $C$. coli is unknown, but it might be possible that $C$. coli is intrinsically more capable of acquiring macrolide resistance.

The overall prevalence of phenicol resistance in Campylobacter has been low $(<2 \%)$, but high prevalence was reported in some geographic areas. Zhou et al. (2016) analyzed 203 Campylobacter isolates from stool samples of diarrhea patients collected between 1994 and 2010 in China, and found the overall rate of florfenicol resistance was $31.5 \%$, lowest at $12 \%$ in $1997-$ 1999 and highest at $62 \%$ in 2009-2010. Ma et al. (2014b) profiled 259 Campylobacter isolates derived from a broiler chicken production chain and found the prevalence of florfenicol resistance in C. jejuni $(37.7 \%)$ was significantly higher than that in $C$. coli $(7.8 \%)$. In another study analyzing antibiotic resistance from broiler chickens, the florfenicol resistance rate of C. jejuni $(79.8 \%)$ was found to be much higher than that of $C$. coli (6.4\%) (Li et al., 2017). In the USA, NARMS analyzed 2258 C. jejuni, 925 C. coli, and 7 Campylobacter lari isolates from retail meat collected between 2002 and 2007, and found no resistance to florfenicol (Zhao et al., 2010). In a NARMS 2014 report, all 114 Campylobacter isolates tested were susceptible to florfenicol, and no genes associated with florfenicol resistance were detected. Similarly, no chloramphenicol or florfenicol resistance in C. jejuni isolates was detected in NAHMS Dairy 2002 and 2007 studies (USDA, 2011). However, the most recent study on Campylobacter isolates from feedlot cattle across five different states revealed $10 \%$ of the C. coli isolates were 
resistant to florfenicol (Tang et al., 2017a) indicating the emergence of florfenicol resistance in bovine Campylobacter.

The prevalence rate of gentamicin-resistant Campylobacter was low in most countries (Kashoma et al., 2015, 2016; Nguyen et al., 2016). According to the NARMS surveillance data, the gentamicin resistance rate in Campylobacter was stable and low before 2007 in the USA, especially in C. jejuni. Between 2007 and 2011, gentamicin resistance increased sharply in C. coli from human and chicken sources, rising from 0 to $12 \%$ in human isolates and from 0.7 to $18 \%$ among retail chicken isolates. In China, several reports revealed a much higher gentamicin resistance rate in Campylobacter, especially for these strains isolated from chicken and swine, and in some studies the resistance rate reached above 90\% (Chen et al., 2010; Yao et al., 2017).

Multidrug resistance (MDR) was defined as being resistant to three or more antimicrobial classes, and the most common drugs Campylobacter is resistant to include FQ, macrolides, tetracycline, florfenicol, trimethoprim-sulfamethoxazole (Li et al., 2017; Ma et al., 2017; Szczepanska et al., 2017) A recent study from Thailand revealed that $100 \%$ of C.jejuni and $98.9 \%$ of C. coli isolates from commercial broiler production chains were MDR, respectively, and most C. coli isolates were resistant to FQ, tetracycline, and trimethoprim (Thomrongsuwannakij et al., 2017). In China, 41.9 to $97.6 \%$ of retail chicken isolates exhibited MDR to three or more classes of antimicrobials (Wang et al., 2016; Li et al., 2017; Ma et al., 2017). Usually, the overall MDR rate in C. coli tends to be higher than in C. jejuni (Li et al., 2017; Ma et al., 2017; Szczepanska et al., 2017). In the USA, the most common MDR pattern was to ciprofloxacin, nalidixic acid, and tetracycline. Except for FQs and tetracycline, the Campylobacter isolates examined are generally susceptible to other antimicrobials, such macrolide, florfenicol, gentamicin and telithromycin (Benoit et al., 2014; Ricotta et al., 2014). In our previous study, we observed $\sim 30 \%$ of $C$. jejuni and $50 \%$ of $C$. coli isolates were resistant to both FQs and tetracycline, respectively, but the MDR rate in C.jejuni and in C. colionly account for 0.3 and $4.3 \%$, respectively (Tang et al., 2017c).

\section{Mechanisms of antibiotic resistance in Campylobacter}

Campylobacter has developed various mechanisms to counteract the selection pressure from antimicrobial agents. These mechanisms include (i) restricting the access of antibiotics to their targets, which involves reducing membrane permeability and increasing extrusion of antibiotics by efflux pumps; (ii) modification or protection of antibiotic targets; and (iii) modification or inactivation of antibiotics. These mechanisms may act together in the resistance to different classes of antibiotic. In this section, mechanisms involved in Campylobacter resistance to FQ, macrolides and florfenicol will be discussed due to their clinical significance or importance for animal production.

\section{FQ resistance mechanisms}

The quinolones are a class of broad spectrum antimicrobials that are potent against both gram-negative and gram-positive bacteria (Andersson and MacGowan, 2003). According to their spectrum of activity, quinolones have been classified into four generations. The majority of quinolones currently used for clinical therapies are FQs, which are derived from the quinolones by a fluorine substitution at the C-6 or C-7 position, thereby increasing their activity against gram-negative bacteria (Andersson and MacGowan, 2003). Once inside bacterial cells, FQ antimicrobials exert their antibacterial effect by interacting with DNA gyrase and topoisomerase IV, resulting in double-stand DNA breaks and cell death (Jacoby, 2005). Two main mechanisms of resistance to FQs are currently recognized in Campylobacter bacteria, including mutations that change the antibiotic's target and that reduce antibiotic intracellular accumulation. In other gram-negative bacteria, target protection mediated by the Qnr protein was also involved in FQ resistance (Martin-Gutierrez et al., 2017), but this mechanism has not been reported in Campylobacter.

In gram-negative bacteria, gyrase is the main target of FQ antibiotics, whereas, in gram-positive bacteria, topoisomerase IV is more susceptible to the action of FQ (Jacoby, 2005). Both enzymes consisting of two pairs of subunits, named GyrA and GyrB (DNA gyrase), and ParC and ParE (topoisomerase IV) (Payot et al., 2006). Although most bacteria have both enzymes, Campylobacter lacks the parC and parE genes and thus they are not the targets of FQ antimicrobials in Campylobacter (Bachoual et al., 2001; Payot et al., 2002; Piddock et al., 2003). Additionally, no mutations in gyrB have been associated with FQ resistance in Campylobacter (Bachoual et al., 2001). Therefore, mutations linked to FQ resistance in C. jejuni and C. coli mainly occur in GyrA. Specifically, resistance to FQs involves amino acid substitutions in a region of the GyrA termed the 'quinolone-resistance-determining region'. This region is located within the DNA-binding domain on the surface of DNA gyrase and corresponding amino acids spans from position 51 to position 106 (E. coli numbering), with common mutations at amino acid positions 83 and 87 (position 86 and 90 in Campylobacter) (Friedman et al., 2001). The most frequent mutation observed in FQ-resistant Campylobacter isolates is Thr-86-Ile, followed by Asp-90-Asn, Thr-86-Lys, Thr-86-Ala, Thr-86-Val, Asp-90-Tyr, and Ala-70-Thr (Wang et al., 1993; Engberg et al., 2001; Luo et al., 2003). The Thr-86-Ile mutation confers a high level of FQ resistance [ciprofloxacin minimum inhibitory concentration (MIC) $\geq 16 \mu \mathrm{g} \mathrm{ml}^{-1}$ ] in Campylobacter, while other mutations are associated with a low to medium level of resistance (MIC $=1-8 \mu \mathrm{g} \mathrm{ml}^{-1}$ ) (Luo et al., 2003; Payot et al., 2006; Yan et al., 2006). Double mutations including Thr-86-Ile/Pro-104-Ser and Thr-86-Ile/Asp-90-Asn have also been linked to FQ resistance in Campylobacter (Payot et al., 2006). Additionally, acquisition of high-level FQ resistance in Campylobacter does not require stepwise accumulation of point mutations in gyrA. Instead, a single point mutation in gyrA can lead to clinically relevant levels of resistance to FQ antimicrobials (Gootz and Martin, 1991; Wang et al., 1993; Ruiz et al., 1998; Luo et al., 2003; Yan et al., 2006).

The CmeABC efflux pump contributes significantly to both intrinsic and acquired resistance of $C$. jejuni to FQ antimicrobials by reducing the accumulation of FQs in Campylobacter cells 
(Ge et al., 2005). In wild type 81-176, inactivation of CmeB led to a 8 -fold reduction in the MIC of ciprofloxacin, suggesting that $\mathrm{CmeABC}$ contributes to the intrinsic resistance of Campylobacter to FQs (Lin et al., 2002). Even in the presence of resistance-conferring GyrA mutations, insertional mutagenesis of $\mathrm{CmeABC}$ led to drastic reduction in ciprofloxacin MIC in $\mathrm{FQ}^{\mathrm{R}}$ isolates, indicating the importance of $\mathrm{CmeABC}$ in $\mathrm{FQ}$ resistance (Luo et al., 2003). Overexpression of CmeABC, either by inactivating its repressor $\mathrm{CmeR}$ or mutating the promoter region of cme $A B C$, increased the resistance to $F Q s$ in Campylobacter (Lin et al., 2005a; Yao et al., 2016). The recently identified CmeABC variant (RE-CmeABC) showed a much higher efficiency in the efflux function and conferred an exceedingly high-level resistance (ciprofloxacin MIC $\geq 256 \mu \mathrm{g} \mathrm{ml}^{-1}$ ) to FQs in the presence of GyrA mutations (Yao et al., 2016). The RE-CmeABC appears to be increasingly prevalent in China, where FQs have been widely used for animal production practices (Yao et al., 2016). By reducing the intracellular concentration of antibiotics, $\mathrm{CmeABC}$ facilitates and promotes the emergence of $\mathrm{FQ}^{\mathrm{R}}$ Campylobacter under selection pressure because GyrA mutations alone are not sufficient to survive the killing effect of ciprofloxacin (Yan et al., 2006). In the absence of a functional CmeABC, many spontaneous gyrA mutants would not be able to emerge under antibiotic selection (Yan et al., 2006).

\section{Macrolide resistance mechanisms}

Macrolide antibiotics, such as erythromycin, azithromycin, clarithromycin, and relithromycin, are a class of natural products that consist of a large macrocyclic lactone ring, which are usually 14-, 15-, or 16-membered (Tenson et al., 2003). Macrolides inhibit protein synthesis by binding to the ribosome that includes $23 \mathrm{~S}$ rRNA and ribosomal proteins. Macrolides are usually used for the treatment of gram-positive cocci (mainly staphylococci and streptococci), gram-positive bacilli, gramnegative cocci, and some gram-negative bacilli, such as Campylobacter and belicobacter (Leclercq, 2002). For clinical therapy of campylobacteriosis, macrolides such as erythromycin are often considered the drug of choice. Three mechanisms have been reported for macrolide resistance in bacteria, which include (i) modification of target sites by mutation or methylation, (ii) active efflux of antibiotics from bacterial cells, and (iii) antibiotic inactivation. In Campylobacter, the first two mechanisms have been documented, but macrolide inactivation by the action of esterases or phosphotransferases has not been reported.

In Campylobacter, positions 2074 and 2075 of the 23S rRNA correspond to positions 2058 and 2059 in E. coli, respectively. These two nucleotides interact directly with macrolide antibiotics and mutations in the two sites impair the binding of macrolides to 23S rRNA (Tenson et al., 2003). To date, four types of point mutations at $23 \mathrm{~S}$ rRNA have been linked to macrolide resistance in Campylboacter, including A2074C, A2074G, A2074T, and A2075G. Among these point mutations, A2075G has been observed most frequently (Jensen and Aarestrup, 2001; Vacher et al., 2003, 2005). C. jejuni and C. coli have three copies of $23 \mathrm{~S}$ rRNA ( $r m$ operon). In most clinical strains that are highly resistant to erythromycin (MIC $>128 \mu \mathrm{g} \mathrm{ml}^{-1}$ ), all three copies of the rrn operons were mutated (Jensen and Aarestrup, 2001; Niwa et al., 2001; Gibreel et al., 2005). When the A2074T mutation occurred only in some of the rrn operons, it only conferred a low level resistance to macrolide (Vacher et al., 2005). However, when the A2074T mutations happened in all three copies of $23 \mathrm{~S}$ rRNA genes, the mutant strains were highly resistant to macrolide (Ohno et al., 2016).

Modification of the ribosomal protein L4 and L22 has also been found conferring macrolide resistance in Campylobacter. L4 and L22 were encoded by the rplD and rplV genes, respectively, and both were considered as a portion of the peptide exit tunnel of the $50 \mathrm{~S}$ ribosome. Amino acids spanning positions 63-74 are reported to be the most important target regions of the L4 protein (Corcoran et al., 2006). Mutation in this region had been reported in several bacteria with high levels of erythromycin resistance (Chittum and Champney, 1994; Tait-Kamradt et al., 2000; Malbruny et al., 2002). In Campylobacter, the G74D modification alone was found to confer low to medium resistance to macrolides (Cagliero et al., 2006). Outside the 63-74 amino acid region of L4, several other amino acid substitutions were associated with macrolide resistance in both Campylobacter and Streptococcus (Doktor et al., 2004; Corcoran et al., 2006). The L22 modifications, including insertion, mutation, and deletion are also involved in macrolide resistance in Campylobacter. Corcoran et al. (2006) identified a unique A103V substitution in the L22 protein, which was linked to high level erythromycin resistance in both C. jejuni and C. coli. Three to four amino acid insertions at position 86 or 98 of the L22 protein were also observed in macrolide-resistant isolates (Caldwell et al., 2008; Lehtopolku et al., 2011).

Recently, a new mechanism of macrolide resistance in Campylobacter has emerged (Qin et al., 2014), which is mediated by the $\operatorname{erm}(B)$ gene that encodes a rRNA methyltransferase. This enzyme adds a methyl group to the A2058 (E. coli numbering) position located within a conserved region of domain $\mathrm{V}$ of the $23 \mathrm{~S}$ rRNA. Methylation at this site gives rise to crossresistance to macrolide, lincosamide, and streptogramin $\mathrm{B}$ $\left(\mathrm{MLS}_{\mathrm{B}}\right.$ phenotype). To date, $43 \mathrm{erm}$ (erythromycin ribosome methylase) genes have been reported (http://faculty.washington.edu/marilynr/), but only $\operatorname{erm}(B)$ has been detected in Campylobacter, including C. jejuni and C. coli in China and Europe (Qin et al., 2014; Deng et al., 2015; Florez-Cuadrado et al., 2016). In the first report of erm(B) in C. coli, it was identified in a $12,035 \mathrm{bp}$ genomic segment on the chromosome and was found to confer a high-level resistance to erythromycin (MIC $512 \mu \mathrm{g} \mathrm{ml}^{-1}$ ). This segment contained 17 open reading frames (ORFs), 8 of which were antibiotic resistance determinants, including erm $(B)$, tet $(O)$, and 6 genes encoding aminoglycoside-modifying enzymes (Qin et al., 2014). Thus, the genomic segment was named as the MDR genomic island (MDRGI). This MDRGI can be transferred between C. jejuni and C. coli via natural transformation (Qin et al., 2014). The erm (B) gene was also later identified in C. jejuni, where it was associated with several antimicrobial resistance genes $[\operatorname{tet}(O)$, aadE and $a a d 9$ ] in a MDRGI that was inserted in the chromosome at a different location when compared with that in C. coli 
(Deng et al., 2015). In Europe, the identified erm(B) in C. coli was also located in a MDRGI, but the MDGRI contents were different from those found in China (Florez-Cuadrado et al., 2016). Interestingly, the erm(B)-carrying MDRGIs have different G+ $C$ contents from the rest of the chromosome, which suggests that Campylobacter acquired erm(B) from other bacterial organisms via horizontal gene transfer (Florez-Cuadrado et al., 2016). The emergence of $\operatorname{erm}(B)$ in Campylobacter is alarming because it alone confers a high-level resistance to macrolide antibiotics and is horizontally transferable. Thus, the spread of erm(B)-positive Campylobacter should be continuously monitored.

The multidrug efflux pump CmeABC also contributes significantly to macrolide resistance in Campylobacter. This was first demonstrated by an insertional mutation of the $c m e B$ gene in wild-type 81-176, which resulted in a 4-fold decrease in the MIC of erythromycin (Lin et al., 2002). Additionally, overexpression of $\mathrm{CmeABC}$ by mutating the $\mathrm{CmeR}$ repressor led to 4 -fold increase in the resistance to erythromycin (Lin et al., 2005a). Even in the highly resistant strains (harboring the resistanceconferring mutations in the $23 \mathrm{~S} \mathrm{rRNA}$ ), inactivating the $\mathrm{cmeB}$ gene led to a drastic reduction in the MIC of erythromycin (Cagliero et al., 2005; Lin et al., 2007) suggesting that the point mutations in $23 \mathrm{~S}$ rRNA and $\mathrm{CmeABC}$ function synergistically in conferring high-level macrolide resistance.

\section{Florfenicol resistance mechanisms}

Florfenicol is a fluorinated derivative of thiamphenicol and has only been used in veterinary medicine since its introduction in the mid-1990s (Syriopoulou et al., 1981). In the USA, florfenicol is currently indicated for the treatment of bovine respiratory disease and bovine interdigital phlegmon. Florfenicol has a broad antibacterial spectrum against both gram-positive and gramnegative organisms, and shows a good tissue penetration property due to its lipophilicity (Schwarz et al., 2004). Once in a bacterial cell, florfenicol binds to the peptidyltransferase center to prevent the peptide chain elongation, resulting in inhibition of protein synthesis and bacterial death. Over the years, bacteria have developed mechanisms to counteract the selection pressure from florfenicol, including (i) modification or protection of the antibiotic targets and (ii) decrease of intracellular concentration by reducing the permeability and increasing efflux.

Functioning as an rRNA methyltransferase, Cfr adds a methyl group at position A2503 of $23 \mathrm{~S}$ rRNA and plays an important role in bacterial resistance to florfenicol (Kehrenberg et al., 2005). Given that position A2503 of $23 \mathrm{~S}$ rRNA is located at the peptidyl transferase center, which is the target of a number of antimicrobial agents, modification of this position affects binding of multiple classes of antibiotics. Indeed, antimicrobial susceptibility testing revealed that Staphylococcus aureus and E. coli strains expressing the $c f r$ gene showed resistance to five chemically distinct classes of antimicrobials, including phenicols, lincosamides, oxazolidinones, pleuromutilins, and streptogramin A (known as the PhLOPS A phenotype) (Long et al., 2006). The Cfr-mediated resistance to oxazolidinones is especially alarming as this class of antibiotics is considered the last resort for the treatment of MDR gram positives (French, 2001). The $c f r$ gene was first discovered on a $16.5-\mathrm{kb}$ plasmid from $S$. sciuri isolate of bovine origin in 2000 (Schwarz et al., 2000). Since its first discovery, $c f r$ has been detected in a number of Gram-positive and Gram-negative bacteria (Schwarz et al., 2000; Dai et al., 2010; Wang et al., 2012a, b; Liu et al., 2013). The cfr gene is often carried by transferable plasmids with additional antibiotic resistance genes, facilitating its dissemination and emergence in different bacterial species and under various selective conditions (Wang et al., 2013; Liu et al., 2014; Li et al., 2015; Zhang et al., 2015). Later, a cfr-like variant, named $c f r(\mathrm{~B})$, was discovered in a mobile genetic element in both Peptoclostridium difficile and Enterococcus faecium of human origin (Deshpande et al., 2015; Hansen and Vester, 2015). Cfr(B) shares $74.9 \%$ amino acid (aa) sequence identity with the original $\mathrm{Cfr}$ and confers the same MDR phenotype (Deshpande et al., 2015).

Although florfenicol is not used for treating Campylobacter infection, its use in animal production imposes a selection pressure on Campylobacter. Recently, a novel $c f r$-like gene, named $c f r$ (C), was identified in Campylobacter coli and Clostridium difficile (Candela et al., 2017; Tang et al., 2017a). In Campylobacter, cfr (C) was located on a conjugative plasmid of $\sim 48 \mathrm{~kb}$ (Tang et al., 2017b) and encodes a 379 aa protein that only shows 55.1 and $54.9 \%$ aa identity to the original Cfr from Staphylococcus sciuri (Schwarz et al., 2000) and the recently reported Cfr(B) from E. faecium, respectively (Deshpande et al., 2015). Cloning of $c f r(C)$ into C. jejuni NCTC11168 and conjugative transfer of the $c f r(\mathrm{C})$-containing plasmid confirmed its role in conferring resistance to phenicols, lincosamides, pleuromutilins, and oxazolidinones, which resulted in 8- to 256-fold increase in their MICs in both C. jejuni and C. coli. These findings established $\operatorname{cfr}(C)$ as a novel MDR gene and represent the first report of a $c f r$-like gene in a foodborne pathogen. In Clostridium difficile, $\quad c f r(C)$ is located on a putative 24 kb-transposon and also confers resistance to $\mathrm{PhLOPS}_{\mathrm{A}}$ (Candela et al., 2017). In addition to Cfr, mutation in the antibiotic target also confers resistance to florfenicol. For example, a G2073A mutation in all three copies of the 23S rRNA was shown to mediate resistance to chloramphenicol and florfenicol in C. jejuni (Ma et al., 2014a).

The typical CmeABC in C. jejuni NCTC 11168 had limited effect on florfenicol resistance (Tang et al., 2017b). However, the recently identified 'super' efflux pump variant, $\mathrm{RE}-\mathrm{CmeABC}$, is much more potent in conferring resistance to florfenicol and other antibiotics (Yao et al., 2016). The Re-CmeABC was discovered from MDR C. jejuni isolates, and transfer of this efflux mechanism to different $C$. jejuni strains resulted in a $>32$-fold increase in the MIC of florfenicol, suggesting its powerful role in the extrusion of florfenicol.

The floR gene, encoding a MDR efflux pump, mediates resistance to chloramphenicol and florfenicol (Arcangioli et al., 1999). It was first been discovered in Salmonella typhimurium DT104 (Arcangioli et al., 1999) and had also been detected in C. coli (Frye et al., 2011). floR encodes a protein of 404 amino acids, which functions as efflux transporter. Interestingly, pp-flo, florSt, flo, and floR, are closely related even though they were assigned different names in the literature (Kim and Aoki, 
1996; Arcangioli et al., 1999; Bolton et al., 1999). Functionally, they all confer resistance to both chloramphenicol and florfenicol. Sequence alignment showed $96-100 \%$ identity in their nucleotide sequences and $88-100 \%$ identity in the amino acid sequences. The fex $A$ and fexB genes, coding for phenicol specific efflux pumps, also confer resistance to florfenicol. They have been found in Staphylococcus, Bacillus, and Enterococcus, but not in Campylobacter (Dai et al., 2010; Liu et al., 2012; Gomez-Sanz et al., 2013).

\section{Beta-lactam resistance mechanisms}

Beta-lactam antibiotics, such as penicillin, inhibit the growth of bacteria by disrupting peptidoglycan cross-linking during bacterial cell wall biosynthesis. Although beta-lactam antibiotics are not commonly prescribed for the treatment of Campylobacter infection, recent studies have proposed that oral beta-lactam, such as co-amoxiclav, could be an appropriate choice when Campylobacter is resistant to both FQ and macrolides. In Campylobacter, two mechanisms of beta-lactam resistance have been documented. One is the production of beta-lactamase OXA-61 and the other one is the multidrug efflux pump.

Several studies have reported that the majority of Campylobacter isolates were ampicillin resistant, and resistance was more common among $C$. coli isolates than among $C$. jejuni isolates (Li et al., 2007; Griggs et al., 2009; Komba et al., 2015). The genome sequence of $C$. jejuni NCTC 11168 revealed the presence of a putative chromosomally encoded class $\mathrm{D}$ betalactamase (Cj0299) (Parkhill et al., 2000). The corresponding gene in a clinical human isolate GC015 has been functionally characterized and was shown to confer a $\geq 32$-fold increase in the MICs of ampicillin, piperacillin, and carbenicillin in C. jejuni (Alfredson and Korolik, 2005). The expression level of the gene can also modulate the susceptibility of Campylobacter to beta-lactams. For example, a single nucleotide mutation (G-T transversion) in the promoter region of bla $a_{\mathrm{OXA}-61}$ led to overexpression of $b l a_{\mathrm{OXA}-61}$ and consequently $\geq 256$-fold increase in beta-lactam resistance in C. jejuni (Zeng et al., 2014). A mutator phenotype resulting from a single amino acid change (G199W) in MutY increased the mutation frequency of the G-T transversion in the blaxA-61 promoter region and consequently elevated the spontaneous ampicillin resistance mutation frequency in $C$. jejuni (Dai et al., 2015). In addition to OXA-61, other uncharacterized beta-lactamase genes may exist in Campylobacter (Griggs et al., 2009). CmeABC also plays an important role in intrinsic resistance to beta-lactam antibiotics as mutation of $\mathrm{CmeB}$ resulted in 32-fold reduction in ampicillin MIC (Lin et al., 2002).

\section{Tetracycline resistance mechanisms}

Tetracyclines are an important class of antibiotics widely used in both human and animal medicine (Chopra, 2001). This class of antibiotics prevent bacterial growth by inhibiting protein synthesis with interaction of the antibiotics to the ribosomal 30 S subunit (Chopra, 2001). The most important mechanism of resistance to tetracyclines results from acquisition of genetically mobile tetracycline resistance (tet) genes, which encode proteins that either extrude tetracyclines, or confer ribosomal protection (Chopra and Roberts, 2001). In Campylobacter spp., two mechanisms of tetracycline resistance were reported, including (i) ribosomal protection protein tet $(\mathrm{O})$ and (ii) endogenous efflux mediated by $\mathrm{CmeABC}$. $\operatorname{tet}(\mathrm{O})$ is the only teracycline-resistance gene identified in Campylobacter so far. The $\operatorname{Tet}(\mathrm{O})$ protein binds to the tetracycline molecule and promote its release from the target site on the ribosome (Connell et al., 2003). The Tet(O) gene may be located on plasmids or the chromosome. The $\mathrm{G}+\mathrm{C}$ content $(40 \%)$ of tet $(O)$ is higher than that of Campylobacter genomes $(\sim 30 \%)$, suggesting that Campylobacter might have obtained the gene from other bacteria by horizontal gene transfer. The multidrug efflux pump, CmeABC, has been shown to contribute to both intrinsic and acquired resistance to tetracycline (Lin et al., 2002; Gibreel et al., 2007). In the $\mathrm{CmeB}$ mutant stain of 81-176 (harboring tet $(\mathrm{O})$ ), the MIC of tetracycline was decreased by 8 -fold (Lin et al., 2002).

\section{Aminoglycoside resistance mechanisms}

The aminoglycoside antibiotics is a class of broad spectrum antibacterial agents used for the treatment of both Gram-positive and Gram-negative organisms. Aminoglycoside antibiotics exert their antibacterial activity by binding the $30 \mathrm{~S}$ ribosomal subunit, thus disturbing the biosynthesis of proteins (Mingeot-Leclercq et al., 1999). Gentamicin is an important aminoglycoside and is used in human beings for treatment of severe infection, including the systemic infection caused by Campylobacter. Gentamicin is also approved for the prevention of bacterial infection-associated death in young food animals, including day-old chicks and 1to 3- day-old turkey poults. Due to the nephro- and ototoxicity, the consumption of gentamicin has significantly decreased. However, the increasing antimicrobial resistance to newer agents has prompted physicians to reevaluate the use of these old antibiotic compounds (Falagas et al., 2008).

Several mechanisms of gentamicin resistance in Campylobacter have been reported. aac $A 4$ encodes an aminoglycoside 6'-N-acytyltransfearse, confer resistance to aminoglycosides containing purpurosamine ring including gentamicin, and was the first gentamicin resistant gene found in C. jejuni isolates (Lee et al., 2002). The gene, aph (2")-If was identified on a MDR conjugative plasmid from a clinical strain of $C$. jejuni, which was isolated from a US soldier deployed to Thailand (Nirdnoy et al., 2005). Although this gene was initially considered as a bifunctional enzyme and annotated as $\operatorname{acc}\left(6^{\prime}-I e\right) / a p h\left(2^{\prime \prime}-I a\right)$ (also named $a a c A / a p h D)$, later it was confirmed as a monofunctional aminoglycoside kinase and named as aph( $\left.2^{\prime \prime}\right)$-If (Toth et al., 2013). A recent study from China found that $a p h\left(2^{\prime \prime}\right)-I f$ was chromosomally encoded and has become the predominant gentamicin resistance determinant in Campylobacter isolates of chicken and swine origin (Yao et al., 2017). A genomic island containing multiple genes encoding aminoglycoside inactivating enzymes has been detected on transmissible plasmids in C. jejuni as well as in the chromosome of $C$. coli (Nirdnoy et al., 2005; Qin et al., 2012). Another gentamicin resistant gene, $a p h\left(2^{\prime \prime}\right)-I g$, which 
share $28 \%$ amino acid identity with $a p h\left(2^{\prime \prime}\right)-I f$, was detected on a $55 \mathrm{~kb}$ conjugative MDR plasmid that shared 95\% nucleotide sequence identity with a pTet plasmid in Campylobacter (Chen et al., 2013). A recent study identified nine variants of gentamicin resistance genes in Campylobacter isolates from the NARMS program, including $a p h\left(2^{\prime \prime}\right)-I b$, -Ic, -Ig, -If, -If1, -If3 and -Ih, aac $\left(6^{\prime}\right) I e /$ $a p h\left(2^{\prime \prime}\right)-I a$ and $\operatorname{aac}\left(6^{\prime}\right)-I e / a p h\left(2^{\prime \prime}\right)-I f 2$ (Zhao et al., 2015). These recent findings clearly indicate a rising trend of aminoglycoside resistance and the continuous emergence of new gentamicin resistance mechanisms in Campylobacter.

\section{MDR mechanisms}

Different from specific resistance mechanisms conferred by target or antibiotic modification, the multidrug efflux pump confers a broad spectrum of resistance to structurally unrelated antimicrobials. In Campylobacter, two MDR mechanisms have been described including Cfr (described above) and multidrug efflux transporters, among which the RND type of transporters are the most significant for antibiotic resistance. In Campylobacter, $\mathrm{CmeABC}$ and $\mathrm{CmeDEF}$ are the functionally characterized RND-type of efflux systems. However, CmeDEF only contribute moderately to intrinsic resistance, while $\mathrm{CmeABC}$ plays a significant role in both intrinsic and acquired resistance of Campylobacter to different antibiotics (Lin et al., 2002; Akiba et al., 2006; Gibreel et al., 2007). CmeABC is a tripartite multidrug efflux pumps and consists a periplasmic fusion protein (CmeA), an inner membrane efflux transporter $(\mathrm{CmeB})$ and an outer membrane protein (CmeC) (Lin et al., 2002). The three proteins function together to form an intact efflux system that extrudes antibiotics and toxic compounds. $\mathrm{CmeB}$ forms a trimeric structure in the bacterial membrane. A recent study using X-ray crystallography and single-molecule fluorescence resonance energy transfer imaging revealed that the $\mathrm{CmeB}$ transporter undergoes conformational transitions uncoordinated and independent of each other, suggesting a novel transport mechanism where $\mathrm{CmeB}$ protomers function independently within the trimer (Su et al., 2017). The function of CmeABC in antibiotic resistance has been demonstrated in many published studies (Lin et al., 2002; Hannula and Hanninen, 2008; Guo et al., 2010; Mavri and Smole Mozina, 2013). In addition to conferring resistance to antibiotics, CmeABC also plays a significant role in bile resistance and thus is essential for Campylobacter colonization in the intestinal tract (Lin et al., 2003).

The recent discovery of RE-CmeABC further demonstrates the key role of CmeABC in conferring MDR (Yao et al., 2016). The $\mathrm{CmeB}$ of RE-CmeABC is unique and shares only $\sim 80 \%$ amino acid identity with the CmeB in NCTC 11168 and other strains. This efflux variant is much more powerful than the typical $\mathrm{CmeABC}$ in the extrusion of antibiotics. For example, transforming C. jejuni NCTC 11168 with RE-CmeABC showed 32-, 16-, 8-, 4-, and 4-fold increases in the MICs of florfenicol, chloramphenicol, ciprofloxacin, erythromycin, and tetracycline, respectively, compared with the recipient strain NCTC 11168 that has a typical CmeABC (Yao et al., 2016). Notably, Re-CmeABC confers exceedingly high-level resistance to FQs, resulting in a ciprofloxacin $\mathrm{MIC} \geq 256 \mu \mathrm{g} \mathrm{ml}^{-1}$ in FQ-resistant C. jejuni isolates. Re-CmeABC also contributes to enhanced emergence of FQ-resistant mutants under antibiotic selection, and drug accumulation assays confirmed the enhanced efflux function of Re-CmeABC (Yao et al., 2016). Interestingly, RE-CmeABC was found to be much more prevalent in C. jejuni $(\sim 35 \%)$ than in C. coli $(\sim 3 \%)$, and the proportion of C. jejuni harboring Re-CmeABC appeared to be on the rise in China (Yao et al., 2016). This trend is probably driven by the extensive use of antibiotics for animal production in China and suggests a fitness advantage for $C$. jejuni strains carrying RE-CmeABC. The findings on Re-CmeABC also explains why florfenicol resistance is highly prevalent, and more so in C. jejuni than in C. coli in China (described above). Additionally, homologs of Re-CmeABC are found in the GenBank database and are deposited by investigators from different countries, suggesting that RE-CmeABC is not unique to China. The exact mechanism for the enhanced function of Re-CmeABC is unknown, but structural modeling suggested that sequence variations in the drug-binding pocket of $\mathrm{CmeB}$ may enhance its interaction with antibiotics and consequently increase its efflux function (Yao et al., 2016).

The expression of cme $A B C$ is subject to regulation. CmeR, a transcriptional repressor of $c m e A B C$, directly interacts with the cme $A B C$ promotor region and represses the transcription of this operon (Guo et al., 2008; Routh et al., 2009). Insertional mutagenesis of $c m e R$ or point mutation in the binding sites of CmeR abolish the binding of $\mathrm{CmeR}$ to the promoter, releasing the repression and enhancing the expression of cmeABC (Cagliero et al., 2007; Guo et al., 2008). CosR, a response regulator in $C$. jejuni, modulates the oxidative stress response and also plays a role in the repression of cme $A B C$ expression (Hwang et al., 2012; Grinnage-Pulley et al., 2016). cosR is an essential gene in Campylobacter, but knockdown of cosR expression by use of antisense peptide nucleic acid increased the transcriptional levels of cme $A B C$ (Hwang et al., 2012). CosR directly binds to the promoter region of $c m e A B C$, but the binding site is independent of the one bound by $\mathrm{CmeR}$ (Grinnage-Pulley and Zhang, 2015). The fact that CmeABC is regulated by multiple mechanisms indicates that it may respond to multiple signals in the host or environment. For example, bile acids, which are present in the intestinal tract of animals, strongly induce the expression of cme $A B C$ by inhibiting the function of CmeR (Lin et al., 2005b; Gu et al., 2007). This induced expression of CmeABC facilitates Campylobacter adaptation to the intestinal environment as it plays a key role in Campylobacter resistance to bile (Lin et al., 2003). Additionally, Salicylate, a nonsteroidal anti-inflammatory compound, is also shown to induce cme $A B C$ expression by inhibiting binding of $\mathrm{CmeR}$ to the promoter of cme $A B C$ (Shen et al., 2011). These examples illustrate the essential functions of $\mathrm{CmeABC}$ beyond antibiotic resistance.

\section{Conclusing remarks}

As a leading cause of bacterial foodborne illness worldwide, Campylobacter continues to pose a significant threat to food safety and public health. As a foodborne pathogen, Campylobacter is 
exposed to antibiotics used for both animal agriculture and human medicine and has shown an amazing ability to adapt to antibiotic selection pressure. To acquire antibiotic resistance, Campylobacter may mutate the targets of antibiotics, such as the case with FQ and macrolide resistance, or acquire new antibiotic resistance determinants from other bacterial organisms by horizontal gene transfer, such as the case with $\operatorname{erm}(B)$ and $\operatorname{cfr}(C)$. Interestingly, Campylobacter tends to acquire foreign antibiotic resistance genes from Gram-positive organisms instead of other Gram-negative bacteria. The exact reason and how this happens are unclear and remain to be investigated. Notably, a highly potent variant of the CmeABC efflux pump (Re-CmeABC) has emerged in C. jejuni, which confers enhanced resistance to multiple classes of antibiotics, providing a powerful mechanism for Campylobacter to adapt to antibiotic treatments. To survive and adapt in various environments, Campylobacter constantly evolves, and it would not be surprising that new antibiotic resistance mechanisms continue to emerge in this foodborne organism. These emerging mechanisms threaten the usefulness of clinically important antibiotics used for treating human campylobacteriosis. Thus, innovative strategies are needed to mitigate the development and spread of antimicrobial resistant Campylobacter, which should be the focus of future research efforts.

\section{Acknowledgment}

The work conducted in the Zhang laboratory is supported by the National Institute of Allergy and Infectious Diseases (grant no. R01AI118283) and USDA National Institute of Food and Agriculture (grants no. 2012-67005-19614 and no. 2017-68003-26499).

\section{References}

Aarestrup FM and Engberg J (2001). Antimicrobial resistance of thermophilic Campylobacter. Veterinary Research 32: 311-321.

Akiba M, Lin J, Barton YW and Zhang Q (2006). Interaction of $\mathrm{CmeABC}$ and $\mathrm{CmeDEF}$ in conferring antimicrobial resistance and maintaining cell viability in Campylobacter jejuni. The Journal of Antimicrobial Chemotherapy 57: 52-60.

Alfredson DA and Korolik V (2005). Isolation and expression of a novel molecular class D beta-lactamase, OXA-61, from Campylobacter jejuni. Antimicrobial Agents and Chemotherapy 49: 2515-2518.

Allos BM (2001). Campylobacter jejuni infections: update on emerging issues and trends. Clinical Infectious Diseases: An Official Publication of the Infectious Diseases Society of America 32: 1201-1206.

Andersson MI and MacGowan AP (2003). Development of the quinolones. The Journal of Antimicrobial Chemotherapy 51(Suppl. 1): 1-11.

Arcangioli MA, Leroy-Setrin S, Martel JL and Chaslus-Dancla E (1999). A new chloramphenicol and florfenicol resistance gene flanked by two integron structures in Salmonella typhimurium DT104. FEMS Microbiology Letters 174: 327-332.

Bachoual R, Ouabdesselam S, Mory F, Lascols C, Soussy CJ and Tankovic J (2001). Single or double mutational alterations of gyrA associated with fluoroquinolone resistance in Campylobacter jejuni and Campylobacter coli. Microbial Drug Resistance 7: 257-261.

Bardon J, Kolar M, Cekanova L, Hejnar P and Koukalova D (2009). Prevalence of Campylobacter jejuni and its resistance to antibiotics in poultry in the Czech Republic. Zoonoses and Public Health 56: $111-116$.

Benoit SR, Lopez B, Arvelo W, Henao O, Parsons MB, Reyes L, Moir JC and Lindblade K (2014). Burden of laboratory-confirmed Campylobacter infections in Guatemala 2008-2012: results from a facility-based surveillance system. Journal of Epidemiology and Global Health 4: 51-59.

Bolton LF, Kelley LC, Lee MD, Fedorka-Cray PJ and Maurer JJ (1999). Detection of multidrug-resistant Salmonella enterica serotype typhimurium DT104 based on a gene which confers cross-resistance to florfenicol and chloramphenicol. Journal of Clinical Microbiology 37: 1348-1351.

Cagliero C, Mouline C, Payot S and Cloeckaert A (2005). Involvement of the CmeABC efflux pump in the macrolide resistance of Campylobacter coli. The Journal of Antimicrobial Chemotherapy 56: 948-950.

Cagliero C, Mouline C, Cloeckaert A and Payot S (2006). Synergy between efflux pump $\mathrm{CmeABC}$ and modifications in ribosomal proteins L4 and L22 in conferring macrolide resistance in Campylobacter jejuni and Campylobacter coli. Antimicrobial Agents and Chemotherapy 50: 3893-3896.

Cagliero C, Maurel MC, Cloeckaert A and Payot S (2007). Regulation of the expression of the CmeABC efflux pump in Campylobacter jejuni: identification of a point mutation abolishing the binding of the CmeR repressor in an in vitro-selected multidrug-resistant mutant. FEMS Microbiology Letters 267: 89-94.

Caldwell DB, Wang Y and Lin J (2008). Development, stability, and molecular mechanisms of macrolide resistance in Campylobacter jejuni. Antimicrobial Agents and Chemotherapy 52: 3947-3954.

Candela T, Marvaud JC, Nguyen TK and Lambert T (2017). A cfr-like gene $\operatorname{cfr}(\mathrm{C})$ conferring linezolid resistance is common in Clostridium difficile. International Journal of Antimicrobial Agents 50: 496-500.

CDC (2009). Campylobacter jejuni infection associated with unpasteurized milk and cheese - Kansas, 2007. MMWR. Morbidity and Mortality Weekly Report 57: 1377-1379.

CDC (2013). Antibiotic Resistance Threats in the United States. http:// www.cdc.gov/drugresistance/threat-report-2013/.

Cha W, Mosci R, Wengert SL, Singh P, Newton DW, Salimnia H, Lephart P, Khalife W, Mansfield LS, Rudrik JT and Manning SD (2016). Antimicrobial susceptibility profiles of human Campylobacter jejuni isolates and association with phylogenetic lineages. Frontiers in Microbiology 7: 589.

Chen X, Naren GW, Wu CM, Wang Y, Dai L, Xia LN, Luo PJ, Zhang $\mathrm{Q}$ and Shen JZ (2010). Prevalence and antimicrobial resistance of Campylobacter isolates in broilers from China. Veterinary Microbiology 144: 133-139.

Chen Y, Mukherjee S, Hoffmann M, Kotewicz ML, Young S, Abbott J, Luo Y, Davidson MK, Allard M, McDermott P and Zhao S (2013). Whole-genome sequencing of gentamicin-resistant Campylobacter coli isolated from U.S. retail meats reveals novel plasmid-mediated aminoglycoside resistance genes. Antimicrobial Agents and Chemotherapy 57: 5398-5405.

Chittum HS and Champney WS (1994). Ribosomal protein gene sequence changes in erythromycin-resistant mutants of Escherichia coli. Journal of Bacteriology 176: 6192-6198.

Chopra I (2001). Glycylcyclines: third-generation tetracycline antibiotics. Current Opinion in Pharmacology 1: 464-469.

Chopra I and Roberts M (2001). Tetracycline antibiotics: mode of action, applications, molecular biology, and epidemiology of bacterial resistance. Microbiology and Molecular Biology Reviews: $M M B R$ 65: 232-260.

Connell SR, Trieber CA, Dinos GP, Einfeldt E, Taylor DE and Nierhaus KH (2003). Mechanism of Tet(O)-mediated tetracycline resistance. The EMBO Journal 22: 945-953.

Corcoran D, Quinn T, Cotter L and Fanning S (2006). An investigation of the molecular mechanisms contributing to high-level erythromycin resistance in Campylobacter. International Journal of Antimicrobial Agents 27: 40-45. 
Dai L, Wu CM, Wang MG, Wang Y, Wang Y, Huang SY, Xia LN, Li BB and Shen JZ (2010). First report of the multidrug resistance gene cfr and the phenicol resistance gene fexA in a Bacillus strain from swine feces. Antimicrobial Agents and Chemotherapy 54: 39533955.

Dai L, Muraoka WT, Wu Z, Sahin O and Zhang Q (2015). A single nucleotide change in mutY increases the emergence of antibioticresistant Campylobacter jejuni mutants. The Journal of Antimicrobial Chemotherapy 70: 2739-2748.

Deng F, Wang Y, Zhang Y and Shen Z (2015). Characterization of the genetic environment of the ribosomal RNA methylase gene erm(B) in Campylobacter jejuni. The Journal of Antimicrobial Chemotherapy 70: 613-615.

Deshpande LM, Ashcraft DS, Kahn HP, Pankey G, Jones RN, Farrell DJ and Mendes RE (2015). Detection of a new $c f r$-like gene, $c f r(B)$, in Enterococcus faecium isolates recovered from human specimens in the United States as part of the SENTRY Antimicrobial Surveillance Program. Antimicrobial Agents and Chemotherapy 59: 6256-6261.

Doktor SZ, Shortridge VD, Beyer JM and Flamm RK (2004). Epidemiology of macrolide and/or lincosamide resistant Streptococcus pneumoniae clinical isolates with ribosomal mutations. Diagnostic Microbiology and Infectious Disease 49: 47-52.

Elviss NC, Williams LK, Jorgensen F, Chisholm SA, Lawson AJ, Swift C, Owen RJ, Griggs DJ, Johnson MM, Humphrey TJ and Piddock LJ (2009). Amoxicillin therapy of poultry flocks: effect upon the selection of amoxicillin-resistant commensal Campylobacter spp. The Journal of Antimicrobial Chemotherapy 64: 702-711.

Endtz HP, Ruijs GJ, van Klingeren B, Jansen WH, van der Reyden T and Mouton RP (1991). Quinolone resistance in campylobacter isolated from man and poultry following the introduction of fluoroquinolones in veterinary medicine. The Journal of Antimicrobial Chemotherapy 27: 199-208.

Engberg J, Aarestrup FM, Taylor DE, Gerner-Smidt P and Nachamkin I (2001). Quinolone and macrolide resistance in Campylobacter jejuni and C. coli: resistance mechanisms and trends in human isolates. Emerging Infectious Diseases 7: 24-34.

Falagas ME, Grammatikos AP and Michalopoulos A (2008). Potential of old-generation antibiotics to address current need for new antibiotics. Expert Review of Anti-infective Therapy 6: 593-600.

Florez-Cuadrado D, Ugarte-Ruiz M, Quesada A, Palomo G, Dominguez L and Porrero MC (2016). Description of an erm (B)-carrying Campylobacter coli isolate in Europe. The Journal of Antimicrobial Chemotherapy 71: 841-843.

French G (2001). Linezolid. International Journal of Clinical Practice 55: 59-63.

Friedman SM, Lu T and Drlica K (2001). Mutation in the DNA gyrase A gene of Escherichia coli that expands the quinolone resistancedetermining region. Antimicrobial Agents and Chemotherapy 45: 2378-2380

Frye JG, Lindsey RL, Meinersmann RJ, Berrang ME, Jackson CR, Englen MD, Turpin JB and Fedorka-Cray PJ (2011). Related antimicrobial resistance genes detected in different bacterial species co-isolated from swine fecal samples. Foodborne Pathogens and Disease 8: 663-679.

Gallay A, Prouzet-Mauleon V, Kempf I, Lehours P, Labadi L, Camou C, Denis M, de Valk H, Desenclos JC and Megraud F (2007). Campylobacter antimicrobial drug resistance among humans, broiler chickens, and pigs, France. Emerging Infectious Diseases 13: 259-266.

Ge B, McDermott PF, White DG and Meng J (2005). Role of efflux pumps and topoisomerase mutations in fluoroquinolone resistance in Campylobacter jejuni and Campylobacter coli. Antimicrobial Agents and Chemotherapy 49: 3347-3354.

Gibreel A and Taylor DE (2006). Macrolide resistance in Campylobacter jejuni and Campylobacter coli. The Journal of Antimicrobial Chemotherapy 58: 243-255.

Gibreel A, Kos VN, Keelan M, Trieber CA, Levesque S, Michaud S and Taylor DE (2005). Macrolide resistance in Campylobacter jejuni and
Campylobacter coli: molecular mechanism and stability of the resistance phenotype. Antimicrobial Agents and Chemotherapy 49: 27532759.

Gibreel A, Wetsch NM and Taylor DE (2007). Contribution of the CmeABC efflux pump to macrolide and tetracycline resistance in Campylobacter jejuni. Antimicrobial Agents and Chemotherapy 51: 3212-3216.

Gomez-Sanz E, Kadlec K, Fessler AT, Zarazaga M, Torres C and Schwarz S (2013). A novel fexA variant from a canine Staphylococcus pseudintermedius isolate that does not confer florfenicol resistance. Antimicrobial Agents and Chemotherapy 57: 5763-5766.

Gootz TD and Martin BA (1991). Characterization of high-level quinolone resistance in Campylobacter jejuni. Antimicrobial Agents and Chemotherapy 35: 840-845.

Griggs DJ, Peake L, Johnson MM, Ghori S, Mott A and Piddock LJ (2009). Beta-lactamase-mediated beta-lactam resistance in Campylobacter species: prevalence of Cj0299 (bla OXA-61) and evidence for a novel beta-Lactamase in C. jejuni. Antimicrobial Agents and Chemotherapy 53: 3357-3364.

Grinnage-Pulley T and Zhang Q (2015). Genetic basis and functional consequences of differential expression of the CmeABC efflux pump in Campylobacter jejuni isolates. PloS ONE 10: e0131534.

Grinnage-Pulley T, Mu Y, Dai L and Zhang Q (2016). Dual repression of the Multidrug Efflux Pump CmeABC by CosR and CmeR in Campylobacter jejuni. Frontiers in Microbiology 7: 1097.

Gu R, Su CC, Shi F, Li M, McDermott G, Zhang Q and Yu EW (2007). Crystal structure of the transcriptional regulator $\mathrm{CmeR}$ from Campylobacter jejuni. Journal of Molecular Biology 372: 583-593.

Guo B, Wang Y, Shi F, Barton YW, Plummer P, Reynolds DL, Nettleton D, Grinnage-Pulley T, Lin J and Zhang Q (2008). Cmer functions as a pleiotropic regulator and is required for optimal colonization of Campylobacter jejuni in vivo. Journal of Bacteriology 190: 1879-1890.

Guo B, Lin J, Reynolds DL and Zhang Q (2010). Contribution of the multidrug efflux transporter $\mathrm{CmeABC}$ to antibiotic resistance in different Campylobacter species. Foodborne Pathogens and Disease 7: $77-83$.

Hannula M and Hanninen ML (2008). Effect of putative efflux pump inhibitors and inducers on the antimicrobial susceptibility of Campylobacter jejuni and Campylobacter coli. Journal of Medical Microbiology 57: 851-855.

Hansen LH and Vester B (2015). A cfr-like gene from Clostridium difficile confers multiple antibiotic resistance by the same mechanism as the cfr gene. Antimicrobial Agents and Chemotherapy 59 5841-5843.

Hoge CW, Gambel JM, Srijan A, Pitarangsi C and Echeverria P (1998). Trends in antibiotic resistance among diarrheal pathogens isolated in Thailand over 15 years. Clinical infectious diseases 26: 341-345.

Humphrey TJ, Jorgensen F, Frost JA, Wadda H, Domingue G, Elviss NC, Griggs DJ and Piddock LJ (2005). Prevalence and subtypes of ciprofloxacin-resistant Campylobacter spp. in commercial poultry flocks before, during, and after treatment with fluoroquinolones. Antimicrobial Agents and Chemotherapy 49: 690-698.

Hwang S, Zhang Q, Ryu S and Jeon B (2012). Transcriptional regulation of the CmeABC multidrug efflux pump and the KatA catalase by CosR in Campylobacter jejuni. Journal of Bacteriology 194: 6883-6891.

Jacoby GA (2005). Mechanisms of resistance to quinolones. Clinical Infectious Diseases 41(Suppl. 2): S120-S126.

Jensen LB and Aarestrup FM (2001). Macrolide resistance in Campylobacter coli of animal origin in Denmark. Antimicrobial Agents and Chemotherapy 45: 371-372.

Kashoma IP, Kassem II, Kumar A, Kessy BM, Gebreyes W, Kazwala RR and Rajashekara G (2015). Antimicrobial resistance and genotypic diversity of Campylobacter isolated from pigs, dairy, and beef cattle in Tanzania. Frontiers in Microbiology 6: 1240.

Kashoma IP, Kassem II, John J, Kessy BM, Gebreyes W, Kazwala RR and Rajashekara G (2016). Prevalence and antimicrobial resistance 
of Campylobacter isolated from dressed beef carcasses and Raw milk in Tanzania. Microbial Drug Resistance 22: 40-52.

Kehrenberg C, Schwarz S, Jacobsen L, Hansen LH and Vester B (2005). A new mechanism for chloramphenicol, florfenicol and clindamycin resistance: methylation of $23 \mathrm{~S}$ ribosomal RNA at A2503. Molecular Microbiology 57: 1064-1073.

Kim E and Aoki T (1996). Sequence analysis of the florfenicol resistance gene encoded in the transferable R-plasmid of a fish pathogen, Pasteurella piscicida. Microbiology and Immunology 40: 665-669.

Komba EV, Mdegela RH, Msoffe PL, Nielsen LN and Ingmer H (2015). Prevalence, antimicrobial resistance and risk factors for thermophilic Campylobacter infections in symptomatic and asymptomatic humans in Tanzania. Zoonoses and Public Health 62: $557-568$.

Leclercq R (2002). Mechanisms of resistance to macrolides and lincosamides: nature of the resistance elements and their clinical implications. Clinical Infectious Diseases: An Official Publication of the Infectious Diseases Society of America 34: 482-492.

Lee MD, Sanchez S, Zimmer M, Idris U, Berrang ME and McDermott PF (2002). Class 1 integron-associated tobramycin-gentamicin resistance in Campylobacter jejuni isolated from the broiler chicken house environment. Antimicrobial Agents and Chemotherapy 46: 3660-3664.

Lehtopolku M, Kotilainen P, Haanpera-Heikkinen M, Nakari UM, Hanninen ML, Huovinen P, Siitonen A, Eerola E, Jalava J and Hakanen AJ (2011). Ribosomal mutations as the main cause of macrolide resistance in Campylobacter jejuni and Campylobacter coli. Antimicrobial Agents and Chemotherapy 55: 5939-5941.

Li B, Ma L, Li Y, Jia H, Wei J, Shao D, Liu K, Shi Y, Qiu Y and Ma Z (2016). Antimicrobial resistance of Campylobacter species isolated from Broilers in Live Bird Markets in Shanghai, China. Foodborne Pathogens and Disease 14: 96-102.

Li B, Ma L, Li Y, Jia H, Wei J, Shao D, Liu K, Shi Y, Qiu Y and Ma Z (2017). Antimicrobial resistance of Campylobacter species isolated from Broilers in Live Bird Markets in Shanghai, China. Foodborne Pathogens and Disease 14: 96-102.

Li D, Wu C, Wang Y, Fan R, Schwarz S and Zhang S (2015). Identification of multiresistance gene $c f r$ in methicillin-resistant Staphylococcus aureus from pigs: plasmid location and integration into a staphylococcal cassette chromosome mec complex. Antimicrobial Agents and Chemotherapy 59: 3641-3644.

Li XZ, Mehrotra M, Ghimire S and Adewoye L (2007). Beta-Lactam resistance and beta-lactamases in bacteria of animal origin. Veterinary Microbiology 121: 197-214.

Lin J, Michel LO and Zhang Q (2002). CmeABC functions as a multidrug efflux system in Campylobacter jejuni. Antimicrobial Agents and Chemotherapy 46: 2124-2131.

Lin J, Sahin O, Michel LO and Zhang Q (2003). Critical role of multidrug efflux pump CmeABC in bile resistance and in vivo colonization of Campylobacter jejuni. Infection and Immunity 71: 4250-4259.

Lin J, Akiba M, Sahin O and Zhang Q (2005a). Cmer functions as a transcriptional repressor for the multidrug efflux pump CmeABC in Campylobacter jejuni. Antimicrobial Agents and Chemotherapy 49: 1067-1075.

Lin J, Cagliero C, Guo B, Barton YW, Maurel MC, Payot S and Zhang Q (2005b). Bile salts modulate expression of the CmeABC multidrug efflux pump in Campylobacter jejuni. Journal of Bacteriology 187: $7417-7424$.

Lin J, Yan M, Sahin O, Pereira S, Chang YJ and Zhang Q (2007). Effect of macrolide usage on emergence of erythromycin-resistant Campylobacter isolates in chickens. Antimicrobial Agents and Chemotherapy 51: 1678-1686.

Liu H, Wang Y, Wu C, Schwarz S, Shen Z, Jeon B, Ding S, Zhang Q and Shen J (2012). A novel phenicol exporter gene, fexB, found in enterococci of animal origin. The Journal of Antimicrobial Chemotherapy 67: 322-325.

Liu Y, Wang Y, Schwarz S, Li Y, Shen Z, Zhang Q, Wu C and Shen J (2013). Transferable multiresistance plasmids carrying $c f r$ in
Enterococcus spp. from swine and farm environment. Antimicrobial Agents and Chemotherapy 57: 42-48.

Liu Y, Wang Y, Dai L, Wu C and Shen J (2014). First report of multiresistance gene of $r$ in Enterococcus species casseliflavus and gallinarum of swine origin. Veterinary Microbiology 170: 352-357.

Long KS, Poehlsgaard J, Kehrenberg C, Schwarz S and Vester B (2006). The Cfr rRNA methyltransferase confers resistance to phenicols, lincosamides, oxazolidinones, pleuromutilins, and streptogramin A antibiotics. Antimicrobial Agents and Chemotherapy 50: 2500-2505.

Luangtongkum T, Jeon B, Han J, Plummer P, Logue CM and Zhang Q (2009). Antibiotic resistance in Campylobacter. emergence, transmission and persistence. Future Microbiology 4: 189-200.

Lucey B, Cryan B, O'Halloran F, Wall PG, Buckley T and Fanning S (2002). Trends in antimicrobial susceptibility among isolates of Campylobacter species in Ireland and the emergence of resistance to ciprofloxacin. The Veterinary Record 151: 317-320.

Luo N, Sahin O, Lin J, Michel LO and Zhang Q (2003). In vivo selection of Campylobacter isolates with high levels of fluoroquinolone resistance associated with gyrA mutations and the function of the CmeABC efflux pump. Antimicrobial Agents and Chemotherapy 47: 390-394.

Ma H, Su Y, Ma L, Ma L, Li P, Du X, Golz G, Wang S and Lu X (2017). Prevalence and characterization of Campylobacter jejuni isolated from Retail Chicken in Tianjin, China. Journal of Food Protection 80: 1032-1040.

Ma L, Shen Z, Naren G, Li H, Xia X, Wu C, Shen J, Zhang Q and Wang Y (2014a). Identification of a novel G2073A mutation in $23 \mathrm{~S}$ rRNA in amphenicol-selected mutants of Campylobacter jejuni. PloS ONE 9: e94503.

Ma L, Wang Y, Shen J, Zhang Q and Wu C (2014b). Tracking Campylobacter contamination along a broiler chicken production chain from the farm level to retail in China. International Journal of Food Microbiology 181: 77-84.

Malbruny B, Nagai K, Coquemont M, Bozdogan B, Andrasevic AT, Hupkova H, Leclercq R and Appelbaum PC (2002). Resistance to macrolides in clinical isolates of Streptococcus pyogenes due to ribosomal mutations. The Journal of Antimicrobial Chemotherapy 49: 935-939.

Martin-Gutierrez G, Rodriguez-Martinez JM, Pascual A, RodriguezBeltran J and Blazquez J (2017). Plasmidic qnr genes confer clinical resistance to ciprofloxacin under urinary tract physiological conditions. Antimicrobial Agents and Chemotherapy 61: e02615-16.

Mavri A and Smole Mozina S (2013). Effects of efflux-pump inducers and genetic variation of the multidrug transporter $c m e B$ in biocide resistance of Campylobacter jejuni and Campylobacter coli. Journal of Medical Microbiology 62: 400-411.

McDermott PF, Bodeis SM, English LL, White DG, Walker RD, Zhao S, Simjee S and Wagner DD (2002). Ciprofloxacin resistance in Campylobacter jejuni evolves rapidly in chickens treated with fluoroquinolones. The Journal of Infectious Diseases 185: 837-840.

Mingeot-Leclercq MP, Glupczynski Y and Tulkens PM (1999). Aminoglycosides: activity and resistance. Antimicrobial Agents and Chemotherapy 43: 727-737.

Nachamkin I, Ung H and Li M (2002). Increasing fluoroquinolone resistance in Campylobacter jejuni, Pennsylvania, USA,1982-2001. Emerging Infectious Diseases 8: 1501-1503.

Nguyen TN, Hotzel H, El-Adawy H, Tran HT, Le MT, Tomaso H, Neubauer H and Hafez HM (2016). Genotyping and antibiotic resistance of thermophilic Campylobacter isolated from chicken and pig meat in Vietnam. Gut Pathogens 8: 19.

Nirdnoy W, Mason CJ and Guerry P (2005). Mosaic structure of a multiple-drug-resistant, conjugative plasmid from Campylobacter jejuni. Antimicrobial Agents and Chemotherapy 49: 2454-2459.

Niwa H, Chuma T, Okamoto K and Itoh K (2001). Rapid detection of mutations associated with resistance to erythromycin in Campylobacter jejuni/coli by PCR and line probe assay. International Journal of Antimicrobial Agents 18: 359-364.

Ohno H, Wachino J, Saito R, Jin W, Yamada K, Kimura K and Arakawa Y (2016). A highly macrolide-resistant Campylobacter jejuni 
strain with rare A2074T mutations in 23S rRNA genes. Antimicrobial Agents and Chemotherapy 60: 2580-2581.

Padungton P and Kaneene JB (2003). Campylobacter spp in human, chickens, pigs and their antimicrobial resistance. The Journal of Veterinary Medical Science 65: 161-170.

Papavasileiou E, Voyatzi A, Papavasileiou K, Makri A, Andrianopoulou I and Chatzipanagiotou S (2007). Antimicrobial susceptibilities of Campylobacter jejuni isolates from hospitalized children in Athens, Greece, collected during 2004-2005. European Journal of Epidemiology 22: 77-78.

Parkhill J, Wren BW, Mungall K, Ketley JM, Churcher C, Basham D, Chillingworth T, Davies RM, Feltwell T, Holroyd S, Jagels K, Karlyshev AV, Moule S, Pallen MJ, Penn CW, Quail MA, Rajandream MA, Rutherford KM, van Vliet AH, Whitehead S and Barrell BG (2000). The genome sequence of the food-borne pathogen Campylobacter jejuni reveals hypervariable sequences. Nature 403: 665-668.

Payot S, Cloeckaert A and Chaslus-Dancla E (2002). Selection and characterization of fluoroquinolone-resistant mutants of Campylobacter jejuni using enrofloxacin. Microbial Drug Resistance 8: 335-343.

Payot S, Bolla JM, Corcoran D, Fanning S, Megraud F and Zhang Q (2006). Mechanisms of fluoroquinolone and macrolide resistance in Campylobacter spp. Microbes and infection/Institut Pasteur 8: 1967-1971.

Pezzotti G, Serafin A, Luzzi I, Mioni R, Milan M and Perin R (2003). Occurrence and resistance to antibiotics of Camplobacter jejuni and Campylobacter coli in animals and meat in northeastern Italy. International Journal of Food Microbiology 82: 281-287.

Piddock LJ, Ricci V, Pumbwe L, Everett MJ and Griggs DJ (2003). Fluoroquinolone resistance in Campylobacter species from man and animals: detection of mutations in topoisomerase genes. The Journal of Antimicrobial Chemotherapy 51: 19-26.

Qin S, Wang Y, Zhang Q, Chen X, Shen Z, Deng F, Wu C and Shen J (2012). Identification of a novel genomic island conferring resistance to multiple aminoglycoside antibiotics in Campylobacter coli. Antimicrobial Agents and Chemotherapy 56: 5332-5339.

Qin S, Wang Y, Zhang Q, Zhang M, Deng F, Shen Z, Wu C, Wang S, Zhang J and Shen J (2014). Report of ribosomal RNA methylase gene erm(B) in multidrug-resistant Campylobacter coli. The Journal of Antimicrobial Chemotherapy 69: 964-968.

Ricotta EE, Palmer A, Wymore K, Clogher P, Oosmanally N, Robinson T, Lathrop S, Karr J, Hatch J, Dunn J, Ryan P and Blythe D (2014). Epidemiology and antimicrobial resistance of international travel-associated Campylobacter infections in the United States, 2005-2011. American Journal of Public Health 104: e108-e114.

Routh MD, Su CC, Zhang Q and Yu EW (2009). Structures of AcrR and CmeR: insight into the mechanisms of transcriptional repression and multi-drug recognition in the TetR family of regulators. Biocbimica et biophysica acta 1794: 844-851.

Ruiz J, Goni P, Marco F, Gallardo F, Mirelis B, Jimenez De Anta T and Vila J (1998). Increased resistance to quinolones in Campylobacter jejuni: a genetic analysis of gyrA gene mutations in quinoloneresistant clinical isolates. Microbiology and Immunology 42: 223-226.

Ruiz-Palacios GM (2007). The health burden of Campylobacter infection and the impact of antimicrobial resistance: playing chicken. Clinical Infectious Diseases 44: 701-703.

Scallan E, Hoekstra RM, Angulo FJ, Tauxe RV, Widdowson MA, Roy SL, Jones JL and Griffin PM (2011). Foodborne illness acquired in the United States - major pathogens. Emerging Infectious Diseases 17: $7-15$.

Schwarz S, Werckenthin C and Kehrenberg C (2000). Identification of a plasmid-borne chloramphenicol-florfenicol resistance gene in Staphylococcus sciuri. Antimicrobial Agents and Chemotherapy 44: $2530-2533$.

Schwarz S, Kehrenberg C, Doublet B and Cloeckaert A (2004). Molecular basis of bacterial resistance to chloramphenicol and florfenicol. FEMS Microbiology Reviews 28: 519-542.
Shen Z, Pu XY and Zhang Q (2011). Salicylate functions as an efflux pump inducer and promotes the emergence of fluoroquinoloneresistant Campylobacter jejuni mutants. Applied and Environmental Microbiology 77: 7128-7133.

Shobo CO, Bester LA, Baijnath S, Somboro AM, Peer AK and Essack SY (2016). Antibiotic resistance profiles of Campylobacter species in the South Africa private health care sector. Journal of Infection in Developing Countries 10: 1214-1221.

Sierra-Arguello YM, Perdoncini G, Morgan RB, Salle CT, Moraes HL, Gomes MJ and do Nascimento VP (2016). Fluoroquinolone and macrolide resistance in Campylobacter jejuni isolated from broiler slaughterhouses in southern Brazil. Avian Pathology: Journal of the W.V.P.A 45: 66-72.

Singh S and Mittal A (2016). Transmembrane domain lengths serve as signatures of organismal complexity and viral transport mechanisms. Scientific Reports 6: 22352.

Stanley K and Jones K (2003). Cattle and sheep farms as reservoirs of Campylobacter. Journal of Applied Microbiology 94 (Suppl.): 104S-113S.

Su CC, Yin L, Kumar N, Dai L, Radhakrishnan A, Bolla JR, Lei HT, Chou TH, Delmar JA, Rajashankar KR, Zhang Q, Shin YK and Yu EW (2017). Structures and transport dynamics of a Campylobacter jejuni multidrug efflux pump. Nature Communications 8: 171.

Syriopoulou VP, Harding AL, Goldmann DA and Smith AL (1981). In vitro antibacterial activity of fluorinated analogs of chloramphenicol and thiamphenicol. Antimicrobial Agents and Chemotherapy 19: 294-297.

Szczepanska B, Andrzejewska M, Spica D and Klawe JJ (2017). Prevalence and antimicrobial resistance of Campylobacter jejuni and Campylobacter coli isolated from children and environmental sources in urban and suburban areas. BMC Microbiology 17: 80.

Tait-Kamradt A, Davies T, Appelbaum PC, Depardieu F, Courvalin P, Petitpas J, Wondrack L, Walker A, Jacobs MR and Sutcliffe J (2000). Two new mechanisms of macrolide resistance in clinical strains of Streptococcus pneumoniae from Eastern Europe and North America. Antimicrobial Agents and Chemotherapy 44: 3395-3401.

Tang Y, Dai L, Sahin O, Wu Z, Liu M and Zhang Q (2017a). Emergence of a plasmid-borne multidrug resistance gene $\operatorname{cfr}(\mathrm{C})$ in foodborne pathogen Campylobacter. The Journal of Antimicrobial Chemotherapy 72: 1581-1588.

Tang Y, Dai L, Sahin O, Wu Z, Liu M and Zhang Q (2017b). Emergence of a plasmid-borne multidrug resistance gene $\operatorname{cfr}(\mathrm{C})$ in foodborne pathogen Campylobacter. The Journal of Antimicrobial Chemotherapy 72: 1581-1588.

Tang Y, Sahin O, Pavlovic N, LeJeune J, Carlson J, Wu Z, Dai L and Zhang Q (2017c). Rising fluoroquinolone resistance in Campylobacter isolated from feedlot cattle in the United States. Scientific Reports 7: 494.

Tenson T, Lovmar M and Ehrenberg M (2003). The mechanism of action of macrolides, lincosamides and streptogramin B reveals the nascent peptide exit path in the ribosome. Journal of Molecular Biology 330: 1005-1014.

Thomrongsuwannakij T, Blackall PJ and Chansiripornchai N (2017). A study on Campylobacter jejuni and Campylobacter coli through commercial broiler production chains in Thailand: antimicrobial resistance, the characterization of DNA gyrase subunit A mutation, and genetic diversity by flagellin A gene restriction fragment length polymorphism. Avian Diseases 61: 186-197.

Toth M, Frase H, Antunes NT and Vakulenko SB (2013). Novel aminoglycoside 2"-phosphotransferase identified in a gram-negative pathogen. Antimicrobial Agents and Chemotherapy 57: 452-457.

USDA (2011). Salmonella, Listeria, and Camplobacter on U.S. Dairy Operation, 1996-2007. USDA-APHIS-VS, CEAH Fort Collins, CO \#578.0311.

Vacher S, Menard A, Bernard E and Megraud F (2003). PCR-restriction fragment length polymorphism analysis for detection of point mutations associated with macrolide resistance in Campylobacter spp. Antimicrobial Agents and Chemotherapy 47: 1125-1128. 
Vacher S, Menard A, Bernard E, Santos A and Megraud F (2005). Detection of mutations associated with macrolide resistance in thermophilic Campylobacter spp. by real-time PCR. Microbial Drug Resistance 11: 40-47.

van Boven M, Veldman KT, de Jong MC and Mevius DJ (2003). Rapid selection of quinolone resistance in Campylobacter jejuni but not in Eschericbia coli in individually housed broilers. The Journal of Antimicrobial Chemotherapy 52: 719-723.

Wang Y, Huang WM and Taylor DE (1993). Cloning and nucleotide sequence of the Campylobacter jejuni gyrA gene and characterization of quinolone resistance mutations. Antimicrobial Agents and Chemotherapy 37: 457-463.

Wang Y, He T, Schwarz S, Zhou D, Shen Z, Wu C, Wang Y, Ma L, Zhang Q and Shen J (2012a). Detection of the staphylococcal multiresistance gene cfr in Eschericbia coli of domestic-animal origin. The Journal of Antimicrobial Chemotherapy 67: 1094-1098.

Wang Y, Wang Y, Schwarz S, Shen Z, Zhou N, Lin J, Wu C and Shen J (2012b). Detection of the staphylococcal multiresistance gene $c f r$ in Macrococcus caseolyticus and Jeotgalicoccus pinnipedialis. The Journal of Antimicrobial Chemotherapy 67: 1824-1827.

Wang Y, Li D, Song L, Liu Y, He T, Liu H, Wu C, Schwarz S and Shen $\mathrm{J}$ (2013). First report of the multiresistance gene cfr in Streptococcus suis. Antimicrobial Agents and Chemotherapy 57: 4061-4063.

Wang Y, Dong Y, Deng F, Liu D, Yao H, Zhang Q, Shen J, Liu Z, Gao Y, Wu C and Shen Z (2016). Species shift and multidrug resistance of Campylobacter from chicken and swine, China, 2008-14. The Journal of Antimicrobial Chemotherapy 71: 666-669.

WHO (2017). Global priority list of antibiotic-resistant bacteria to guide research, discovery, and development of new antibiotics Tacconelli-Magrini 25 Feb 2017. http://www.who.int/medicines/ publications/global-priority-list-antibiotic-resistant-bacteria/en/.

Wozniak-Biel A, Bugla-Ploskonska G, Kielsznia A, Korzekwa K, Tobiasz A, Korzeniowska-Kowal A and Wieliczko A (2017). High prevalence of resistance to fluoroquinolones and tetracycline Campylobacter spp. Isolated from Poultry in Poland. Microbial Drug Resistance.

Yan M, Sahin O, Lin J and Zhang Q (2006). Role of the CmeABC efflux pump in the emergence of fluoroquinolone-resistant
Campylobacter under selection pressure. The Journal of Antimicrobial Chemotherapy 58: 1154-1159.

Yao H, Shen Z, Wang Y, Deng F, Liu D, Naren G, Dai L, Su CC, Wang B, Wang S, Wu C, Yu EW, Zhang Q and Shen J (2016). Emergence of a potent multidrug efflux pump variant that enhances Campylobacter resistance to multiple antibiotics. mBio 7: e01543-16.

Yao H, Liu D, Wang Y, Zhang Q and Shen Z (2017). High prevalence and predominance of the $a p h\left(2^{\prime \prime}\right)$-If gene conferring aminoglycoside resistance in Campylobacter. Antimicrobial Agents and Chemotherapy 61: e00112-17.

Zeng X, Brown S, Gillespie B and Lin J (2014). A single nucleotide in the promoter region modulates the expression of the betalactamase OXA-61 in Campylobacter jejuni. The Journal of Antimicrobial Chemotherapy 69: 1215-1223.

Zhang Q, Lin J and Pereira S (2003). Fluoroquinolone-resistant Campylobacter in animal reservoirs: dynamics of development, resistance mechanisms and ecological fitness. Animal Health Research Reviews 4: 63-71.

Zhang R, Sun B, Wang Y, Lei L, Schwarz S and Wu C (2015). Characterization of a cfr-carrying plasmid from porcine Escherichia coli that closely resembles plasmid pEA3 from the plant pathogen Erwinia amylovora. Antimicrobial Agents and Chemotherapy 60: 658-661.

Zhao S, Young SR, Tong E, Abbott JW, Womack N, Friedman SL and McDermott PF (2010). Antimicrobial resistance of Campylobacter isolates from retail meat in the United States between 2002 and 2007. Applied and Environmental Microbiology 76: 7949-7956.

Zhao S, Mukherjee S, Chen Y, Li C, Young S, Warren M, Abbott J, Friedman S, Kabera C, Karlsson M and McDermott PF (2015). Novel gentamicin resistance genes in Campylobacter isolated from humans and retail meats in the USA. The Journal of Antimicrobial Chemotherapy 70: 1314-1321.

Zhou J, Zhang M, Yang W, Fang Y, Wang G and Hou F (2016). A seventeen-year observation of the antimicrobial susceptibility of clinical Campylobacter jejuni and the molecular mechanisms of erythromycin-resistant isolates in Beijing, China. International Journal of Infectious Diseases: IJID: Official Publication of the International Society for Infectious Diseases 42: 28-33. 